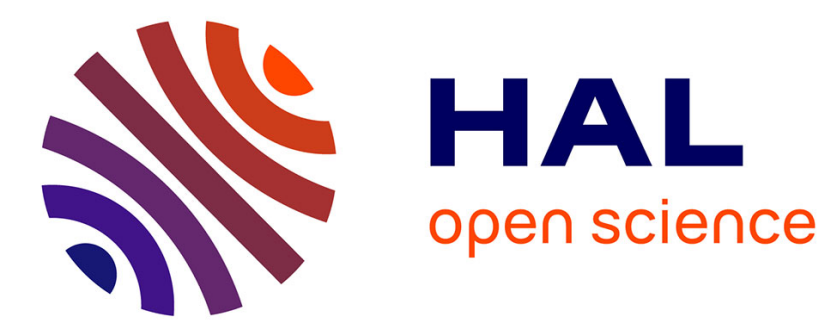

\title{
La réflexivité des acteurs universitaires : un mode d'appropriation par simplification des logiques d'évaluation du NPM ?
}

\author{
Laurent Mériade
}

\section{To cite this version:}

Laurent Mériade. La réflexivité des acteurs universitaires : un mode d'appropriation par simplification des logiques d'évaluation du NPM ?. @GRH, 2013, 9 (4), 10.3917/grh.134.0067 . hal-01507569

\author{
HAL Id: hal-01507569 \\ https://hal.science/hal-01507569
}

Submitted on 10 Mar 2021

HAL is a multi-disciplinary open access archive for the deposit and dissemination of scientific research documents, whether they are published or not. The documents may come from teaching and research institutions in France or abroad, or from public or private research centers.
L'archive ouverte pluridisciplinaire HAL, est destinée au dépôt et à la diffusion de documents scientifiques de niveau recherche, publiés ou non, émanant des établissements d'enseignement et de recherche français ou étrangers, des laboratoires publics ou privés. 


\title{
La réflexivité des acteurs universitaires : un mode d'appropriation par simplification des logiques d'évaluation du NPM?
}

\author{
Laurent Mériade \\ IAE Clermont Auvergne - CleRMA- Chaire « santé et territoires »
}

\begin{abstract}
Résumé
Les transformations qui s'opèrent au sein des universités françaises depuis le début des années 2000 témoignent du succès du concept du "New Public Management" (NPM) dans les organisations publiques (Vinokur, 2008) que les réformes récentes (L.O.L.F. en 2006 et L.R.U. en 2007) sont venues concrétiser.

Notre préoccupation théorique est de questionner le processus d'appropriation de l'instrumentation du NPM (Lascoumes et Simard, 2011) en associant l'approche par les leviers de contrôle (Simons 1995) et la logique d'appropriation des outils de gestion (de Vaujany et Grimand 2005).

De manière pratique, nous étudions les perceptions de la performance des présidents d'université afin de mesurer leur contenu réflexif (Archer, 1995 ; 2002) à partir d'une étude approfondie des principaux indicateurs d'évaluation complétée par une enquête exploratoire.

Trois niveaux de simplification des instruments d'évaluation sont identifiés et rattachés à trois niveaux d'appropriation combinant rationalité et réflexivité des agents publics.
\end{abstract}

Mots clés : évaluation de la performance, NPM, universités, appropriation, réflexivité, simplifications.

\begin{abstract}
The changes taking place in the french universities since the early 2000s demonstrate the success of the concept of "New Public Management" (NPM) in public organizations (Vinokur, 2008) that the recent reforms (L.O.L.F. in 2006 and L.R.U. 2007) came to realize.

Our theoretical concern is to question the process of appropriation of the NPM instrumentation (Lascoumes and Simard, 2011) by combining the approach of the levers of control (Simons 1995) and the logic of appropriation of tools management (Vaujany and Grimand 2005). Practically, we study the perception of the performance by the university presidents to measure their reflexive content (Archer, 1995; Bhaskar 1989) from a detailed study of the main indicators of evaluation completed by an exploratory survey.

Three levels of simplification of assessment tools are identified and associated with three levels of appropriation combining rationality and reflexivity of public officials.
\end{abstract}

Keywords: performance evaluation, NPM, universities, appropriation, reflexivity, simplifications. 
En matière de management, les simplifications des outils et normes de gestion ont, à toute époque, été à la fois un problème et une solution aux exigences de la prise de décision dans les organisations. Elles sont souvent destinées à réduire les tensions entre le possible et le réalisable, entre l'espérance et la peur de tout perdre (Green, 2010). Confrontés à des situations complexes, les managers ont tendance à recourir immédiatement à des solutions de management sans avoir cerné les problèmes auxquels elles sont censées répondre. Pour de Geuser et Fiol (2003), cette dialogique entre simplification et complexité a toujours constitué un des principaux défis du management et plus spécifiquement de la gestion publique.

Avec notamment la L.O.L.F. (Loi Organique relative aux Lois de Finances), en 2006, et la L.R.U. (Loi relative aux Libertés et Responsabilités des Universités), en 2007, les universités françaises sont passées d'une logique de moyens à une logique de résultat (Vinokur, 2008) en mobilisant souvent l'outillage managérial du New Public Management (NPM). Elles se retrouvent au centre de tensions entre complexité et décision pour lesquelles les simplifications des modes de management constituent souvent des solutions pragmatiques privilégiées.

A travers l'analyse de la supposée homogénéité de ces simplifications apportées à l'évaluation de la performance universitaire, notre préoccupation est de questionner le processus d'appropriation de l'instrumentation du NPM (Lascoumes et Simard, 2011). L'enjeu de cet article renvoie à la problématique plus générale de l'instrumentation managériale des organisations publiques articulée par le NPM, pour l'essentiel, autour de la performance financière (Martinet, 2008). Le développement des indicateurs de performance dans les universités françaises s'apparentant plus à un emprunt d'artefacts managériaux qu'à une véritable institutionnalisation des valeurs du NPM (Mazouz et al. 2012).

En associant, dans notre approche, la théorie de l'appropriation (de Vaujany et Grimand 2005) et le dualisme analytique d'Archer (1995) nous portons notre attention sur la réflexivité des acteurs de l'Université et sur son intérêt en vue d'une meilleure compréhension des adaptations opérées face aux logiques d'évaluation de la performance du NPM. De Vaujany (2005) concentre son attention sur les modes d'appropriation des mécanismes de contrôle et d'évaluation en tant que « processus de régulations individuelle ou collective qui s'inscrit dans la durée ». Pour cela, l'auteur distingue trois formes d'appropriation successives (valeur-caution, valeur d'assimilation et valeur «à l'usage ») que nous cherchons à rapprocher des simplifications des modes de management de la performance publique dans les universités françaises.

L'enjeu de notre article n'est pas de remettre en cause l'existence de ces simplifications mais au contraire de mieux les cerner afin de les rendre visibles et identifiables aux yeux des contrôleurs et 
des décideurs. Il s'agit d'insister sur le caractère simplificateur des modes de management public là où, la conscience individuelle des dirigeants aurait tendance à favoriser l'oubli de ces simplifications.

Nous proposons une lecture « réflexive » de la substance de ces simplifications nous permettant de décrire un processus d'appropriation par simplification des indicateurs de la performance dans les universités françaises. Nous étudions la performance, au sens de Pesqueux (2005), comme l'accomplissement d'un processus ou d'une tâche avec les résultats qui en découlent et le succès que l'on peut y attribuer.

Le principal intérêt théorique de notre recherche est de s'interroger sur le processus d'appropriation par simplification de l'usage des indicateurs de la performance en analysant les diverses formes que ces simplifications peuvent prendre. Nous nous intéressons plus particulièrement ici à l'évaluation de la performance en tant qu'outils de gestion simplifiés qui véhiculent des croyances, des valeurs et des normes dont les limites sont initialement déterminées par le choix de l'instrument. Ce développement d'outils simplificateurs voire «simplistes » au nom du « réalisme » de la pensée rationaliste ou néo-rationaliste (Simon, 1947) a eu pour avantage de favoriser la mise en œuvre à grande échelle des systèmes d'évaluation de la performance dans les organisations publiques et notamment dans les universités.

La libéralisation des bureaucraties inspirée par le NPM (Giauque, 2003) a permis le déploiement de ces outils d'évaluation mais ceci s'est souvent réalisé comme «allant de soi » sans interrogation approfondie ou feed back sur les modes de construction de ces instruments. C'est ce retour sur les fondements simplificateurs des outils d'évaluation de la performance inspirés par le NPM que nous voulons entamer pour mettre en évidence l'hétérogénéité de ces simplifications et leur utilité dans les processus d'appropriation de ces outils. Mazouz et al. (2012), en analysant ces outils comme des artefacts managériaux ouvre un large champ d'étude théorique et pratique des effets et impacts de la transposition des instruments et pratiques du NPM dans lequel nous souhaitons inscrire les simplifications que nous étudions.

Sur un plan pratique, l'objectif de notre travail est l'étude des intérêts concrets d'une meilleure connaissance de ces processus simplificateurs et l'analyse de leurs effets sur les systèmes de management de la performance universitaire prescrit par le New Public Management. Nous proposons une lecture «réaliste critique» au sens d'Archer de ces formes d'appropriation par simplification afin de prendre en compte leurs modes et leur rythme d'intervention. Nous aborderons ce problème par une analyse plus spécifique des simplifications des indicateurs d'évaluation de la performance réalisées à la fois par les acteurs externes (autorité de tutelle ou 
législateur) et acteurs internes (présidents d'université).

Sur le plan méthodologique, l'étude des simplifications des indicateurs d'évaluation de la performance des universités a été réalisée tout d'abord à partir d'une analyse des indicateurs d'évaluation utilisés par l'A.E.R.E.S. (Agence d'Évaluation de la Recherche et de l'Enseignement Supérieur) et la D.G.E.S.I.P. (Direction Générale de l'Enseignement Supérieur et de l'Insertion Professionnelle) lors de leurs évaluations. Elle a été complétée par une analyse des représentations de la performance des présidents d'université dans le cadre d'une enquête exploratoire menée auprès des présidents des quatre-vingt-cinq universités françaises.

\section{Le contexte des universités françaises}

Un peu partout dans le monde et plus particulièrement en Europe, l'évaluation de la performance des organismes publics est présentée comme un impératif. Cette injonction, portée notamment par le New Public Management (NPM) développe une marchandisation systématique des services publics (Pesqueux, 2005) décrite comme une réponse à la pression croissante des usagers pour obtenir des services de qualité et individualisés.

En tant qu'opérateurs de l'État, les universités françaises sont soumises aux nouvelles règles de gestion définies par la L.O.L.F. votée en août 2001 et mise en place au 1er janvier 2006. En 2007, la L.R.U. instaurant le passage aux R.C.E. (Responsabilités et Compétences Élargies), entraîne l'obligation de maîtriser la masse salariale, le patrimoine immobilisé et le pilotage budgétaire de l'établissement. En 2009, l'introduction du modèle SYMPA (Système de répartition des Moyens à la Performance et à l'Activité), en remplacement du modèle SAN REMO (Système ANalytique de REpartition de Moyens), interjette une méthode de calcul de dotation à l'activité, proche de celle qui a été implantée dans les établissements publics de santé, la T2A (Tarification A l'Activité).

Ce changement législatif récent, autour des universités françaises, s'inscrit plus globalement dans une mise en concurrence des établissements au niveau mondial (Granget, 2009) constatable dans la multiplication des classements nationaux et internationaux (Classement de Shanghai, du Times Higher Education Supplement, de Webometrics, de l'Ecole des Mines de Paris ou encore ceux de journaux généralistes tels Newsweek, Financial Times ou The Economist pour les plus connus).

A la suite de la déclaration de la Sorbonne (1998) initiée par les principaux pays de l'Union Européenne (Allemagne, France, Italie, Royaume-Uni), le processus de Bologne (1999) a inscrit les universités françaises dans un rapprochement entre enseignement supérieur, recherche et compétitivité économique repris astucieusement par la stratégie européenne de Lisbonne (2000) sur l'économie de la connaissance. 
Ces changements se sont engagés alors qu'un marché international de l'enseignement supérieur se développait (Paradeise, 2012) sous les effets conjugués de la croissance de la scolarisation, de la mondialisation des entreprises et de nouvelles pratiques d'enseignement (elearning, serious games, enseignement inversé). Les réformes initiées en France par la L.O.L.F. et la L.R.U. ont représenté des adaptations au contexte français ne reniant pas les principes de marchandisation et de management de la performance du marché mondial de la connaissance (Musselin, 2001).

Sous couvert d'autonomie, cette approche du management universitaire par la performance est avant tout au service d'une vision financière du fonctionnement des universités et elle n'est pas sans ambiguïté. Bouquin (2004) a d'ailleurs déclaré, à son propos, qu'il s'agit bien «d'une notion ambiguë maniée par des personnages ambigus». Cette ambiguïté provient de la lecture entrepreneuriale (Granget, op.cit.) que l'on peut faire des objectifs de performance où l'université doit renforcer sa fonction d'acteur du développement économique (valorisation de sa recherche et « employabilité » de ses diplômés) tout en conservant des modes de négociation privilégiant les rationalités politique et professionnelle.

Les universités françaises sont des organisations où la complexité, et donc les simplifications, apparaissent de manière magistrale. Ce contexte de recherche nous permet donc d'étudier avec encore plus d'acuité cette problématique de la simplification de la performance et de ses modes d'évaluation. Par contre, si l'on suit Hofstede (1981, 1978), il semble que, dans les bureaucraties professionnelles comme les universités (Mintzberg, 1990), les simplifications les plus visibles soient essentiellement prescrites et servent à un contrôle cybernétique de type diagnostique présentant certaines limites. Les modes de fonctionnement universitaires, mis en lumière notamment par le modèle du « garbage can » dans les « anarchies organisées » (Cohen, March et Olsen 1972, Musselin, 1997), révèlent l'importance des débats qui rythment la vie universitaire au gré de ses diverses instances de délibération (conseils, assemblées, commissions, etc.). Dans les universités françaises, ce sont essentiellement des modes prescrits d'évaluation de la performance qui sont encore à l'œuvre limitant, par leur «endogénéité », les pratiques de dialogue de gestion interne et privilégiant une logique de surveillance (Carassus, Baradat et Dupuy 2011).

En substituant la gestion par objectif et résultats à celle des moyens et des ressources (Mazouz et Coté,2005), la L.O.L.F. a introduit une obligation de performance dans la gestion financière des universités (Mignot-Girard, 2008) selon un mode d'évaluation intégrant des indicateurs de performance d'abord définis par des programmes ministériels et des Projets Annuels de Performance (PAP) spécifiques. Les universités françaises sont essentiellement concernés par deux programmes (Formation et recherche universitaire : programme 150 et Vie étudiante : programme 
231) qui sont chacun déclinés sous la forme de cinq à six objectifs majeurs évalués par des cibles de performance relatives à trois à cinq indicateurs par objectif. Ces indicateurs sont repris et développés par l'A.E.R.E.S. et la D.G.E.S.I.P. dans leurs évaluations pour au total proposer une base de 160 à 200 indicateurs de la performance reprenant les objectifs des deux programmes ministériels.

Les dispositifs d'évaluation sont formalisés par une première vague d'indicateurs caractérisant l'établissement et son évolution pluriannuelle (à 4 ans ou 5 ans selon les organismes d'évaluation). Elle porte, d'une part, sur les effectifs étudiants des universités, le nombre de diplômes au niveau L et $\mathrm{M}$, le nombre de personnel exprimé en ETP (Equivalent temps plein) et, d'autre part, sur des données budgétaires relatives au pourcentage d'exécution des budgets (fonctionnement et investissement) et à la masse salariale et son évolution pluriannuelle.

La deuxième vague d'indicateurs évaluent, sur 4 ou 5 ans, les axes spécifiques d'évaluation identifiés en matière d"enseignement et recherche universitaires. Ils sont au nombre de sept et portent successivement sur :

- la recherche (principaux indicateurs : nombre d'enseignants-chercheurs titulaires, budget de recherche exécuté, nombre de contrats de recherche);

- la valorisation de la recherche (principaux indicateurs: nombre d'emplois crées par des incubateurs, nombre de publications et de dépôts de brevets);

- les formations (principaux indicateurs: taux de réussite en L1 et M2, taux d'étudiants boursiers, taux d'attractivité internationale, taux d'insertion professionnelle);

- la vie étudiante (principaux indicateurs: dépenses en faveur de la vie étudiante, taux d'étudiant participant à des activités culturelles ou sportives) ;

- les relations extérieures (principaux indicateurs: nombre de M2 co-habilités, nombre de collaborations scientifiques inter-établissements, budget des partenariats);

- les relations internationales (principaux indicateurs : part des publications ou brevets cosignés avec un chercheur étranger, nombre d'étudiants ayant obtenu une certification en langue, nombre d'étudiants en mobilité internationale) ;

- la gouvernance (principaux indicateurs : nombre d'agents fonctionnaires catégories A, B et C, taux d'enseignant-chercheurs dans l'effectif enseignant total, part et répartition des heures complémentaires d'enseignement, montant des ressources par étudiant, budget consacré à la restructuration des locaux).

$\mathrm{Au}$ total pour ses sept axes d'évaluation, ce sont 40 domaines d'évaluation qui sont traités pour chacun desquels 4 à 5 indicateurs sont mesurés de manière pluriannuelle et en moyenne afin 
notamment de fournir une valeur cible à atteindre par indicateur. C'est seulement à ce niveau qu'intervient la part principale de négociation entre le ministère de tutelle et les établissements universitaires mais elle est en partie biaisée par le manque de souplesse des indicateurs retenus qui empêchent de les amender ou les modifier en fonction des spécificités de chacune des universités.

Le contexte universitaire français, où ces nouveaux modes d'évaluation de la performance ont laissé peu de place à l'interactivité, devient un terrain d'expression de la réflexivité des gestionnaires et agents publics en mesure de décrire les «contextualisations » et les adaptions des instruments qui se concrétisent, selon nous, par les simplifications des indicateurs de la performance que nous étudions ici.

A l'Université, avec l'élargissement des responsabilités attribuées aux présidents, la multiplication des objectifs de performance, la rapidité des choix à appliquer et le degré d'incertitude élevé génèrent une très forte anxiété. A partir des outils du NPM, les dirigeants d'université recourent à des procédés plus traditionnels et assez peu réflexifs afin de simplifier les situations auxquelles ils doivent faire face en adoptant des attitudes dont, comme nous allons le voir dans les lignes qui suivent, la rationalité mérite d'être interrogée.

\section{国 Le New Public Management face à la réflexivité des acteurs}

\subsection{Le New Public Management en France}

Emery et Giauque (2005) perçoivent la mesure et l'évaluation de la performance publique comme un artefact qui s'est construit dans les interstices ouverts par les postulats élémentaires du New Public Management (logique de résultat, mise en concurrence des services publics, rationalisation des coûts publics). Plutôt qu'évaluer ou mesurer les véritables impacts des politiques et services publics, il fut bien plus «aisé d'analyser et de trouver des indicateurs sur les processus de travail et sur les prestations fournies ». Le NPM a privilégié le contrôle des détails opérationnels plutôt que le pilotage stratégique des services publics. Ceci a limité l'évaluation de la performance à des prescriptions managériales dirigées vers les gestionnaires et agents publics les obligeant à se politiser afin de combler le vide stratégique laissé par cette instrumentalisation de l'évaluation de la performance. Bartoli et al. (2011), en présentant les risques de tensions éthiques générés par l'institutionnalisation ou l'instrumentalisation du NPM, insistent sur leurs effets potentiellement contre-productifs et sur l'apparition de réactions adaptatives de la part des gestionnaires et des agents publics. Une des principales formes de réaction fut de simplifier la performance à partir de la confrontation entre cette nouvelle instrumentalisation de l'évaluation de la performance et leur propre connaissance des impératifs de service public et des attentes des usagers. Cette réaction présente des intérêts pratiques et méthodologiques manifestes en matière d'expertise des impacts du 
NPM car elle se concentre sur les représentations de ces agents et gestionnaires publics et elle peut être analysée comme un processus d'appropriation par simplification des outils d'évaluation de la performance publique.

Le New Public Management construit une tendance de repli de l'administration sur elle-même, déjà entraperçue par Crozier (1963) puis Dupuy (1998), qui privilégie ses propres préoccupations au détriment des résultats à fournir aux usagers. Christensen (1998) voit dans l'arrivée au pouvoir, à l'aube des années 80, en Grande- Bretagne et aux Etats-Unis de Margareth Thatcher et Ronald Reagan, l'avènement synchronisée du NPM et d'une pensée néolibérale en réponse à une conjoncture économique de crise (2ème choc pétrolier) et un accroissement significatif de la dépense publique. Lévesque (2012) insiste sur le caractère très homogène des préceptes du NPM qui lui permet d'exercer encore une grande influence sur les réformes et les innovations dans les administrations publiques. Hood (1991) concentre ces préceptes autour de l'introduction des méthodes et d'outils de gestion du privé dans le secteur public au nom de la performance, la créativité et la prise de risque.

Pour Pfeffer et Sutton (2006), cette tendance à la limitation et à la libéralisation des services publics a été facilitée par un effet d'imitation des outils de gestion privée dans le domaine public sans interrogation préalable sur les convergences et les divergences de ces deux modèles de gestion. Urio (1998) parle de «3E » (Economie, Efficacité, Efficience) pour définir les principes fondateurs du NPM dont la mesure va se concentrer essentiellement sur des variables internes. Pettigrew (1997) va plus loin encore en résumant les objectifs du NPM à quatre objectifs essentiels :

- recentrage du secteur public sur les activités régaliennes,

- autonomie et responsabilisation des agents et gestionnaires publics accordées aux dirigeants et aux échelons intermédiaires (principle of managerialization and principle of accountability),

- négociation des ressources et mise en concurrence des acteurs publics,

- évaluation du rapport ressources/création de valeur (value for money).

Ces principes, en introduisant la rationalité managériale, entrent en opposition avec les fondements de la bureaucratie weberienne (1919) reposant sur une rationalité juridique (système hiérarchisé, expertise des fonctionnaires, impersonnalité des règles). Au centre de cette rationalité managériale se trouvent les notions d'efficacité et de performance qui sont présentées par Lauffer et Burlaud (1980) comme le stade ultime de la légitimation de l'intervention publique lorsque les autres valeurs publiques (éthique, intérêt général, continuité du service) ont perdu une grand part de leur légitimité. Doit-on voir dans les logiques et les outils d'évaluation du NPM essentiellement un acte 
de légitimation et de communication publique ? Ou peut-on les percevoir comme un acte de gestion dont les limites restent encore à fixer?

Les réponses à ces questions restent encore très controversées. Pour certains, le secteur public doit se substituer aux défaillances de la concurrence grâce notamment à l'introduction des outils et méthodes de gestion du secteur privé (Jacot et Fouquet 2007). Pour d'autres, la justification de l'intervention publique doit s'obtenir par l'amélioration de l'intérêt général et les outils d'évaluation publique devront en dépendre.

Pour Bezes (2005), La démarche de NPM la plus aboutie fut celle du Royaume dans les années 80 et 90 et elle donna naissance à un système d'expertise et d'évaluation comportant organismes de contrôle, d'audit, d'évaluation et d'inspection qui ont progressivement dénaturer le service public en lui attribuant de nouvelles fonctions bureaucratiques alors que son intention première reposait sur une recherche de flexibilité. Cet auteur, en étudiant le cas de Royaume Uni, parle du NPM comme un concept non paradigmatique car pas en mesure de couvrir l'ensemble des domaines d'intervention publique produisant des performances. Il s'agit d'une faiblesse forte du NPM, c'est à dire son incapacité à circonscrire l'ensemble de la performance publique alors même qu'il fondait sa raison d'être et sa légitimité sur son universalité.

En France, les idées du NPM se sont imposées beaucoup plus récemment. L'adoption en 2001 de la Loi Organique relative aux Lois de Finances (LOLF) est assez emblématique de cette adhésion (Bezes, 2005, Emery, Giauque 2005, Emery 2008) car elle reprend les logiques de résultat et de contrôle de la performance publique qui doivent légitimer l'autonomie et la responsabilisation des gestionnaires et agents publics.

Pour de Visscher (2004), l'adoption dans la LOLF des principes du NPM a deux conséquences essentielles sur les agents publics : leur plus grande responsabilisation qui induit leur nécessaire politisation. Ces conséquences sont assez visibles dans les universités françaises qui en tant qu'organisation politique diffuse progressivement une logique de compromis et de négociation d'abord auprès des responsables de services ou d'UFR (Unité de Formation et de Recherche) puis vers l'ensemble des acteurs (enseignants, personnels administratifs et techniques voire étudiants). Notre étude de la réflexivité des présidents d'université est destinée à étudier la manière dont ces derniers réinterprètent les logiques du NPM à partir de leur propre expérience et leurs contraintes professionnelles. Cette réflexivité participe grandement à l'appropriation des outils de gestion qui selon nous dans les universités se développe par simplification de la complexité des missions et des indicateurs. Nous nous focalisons sur le domaine de l'évaluation de la performance en vue de décrire l'outil d'évaluation comme un compromis, souvent politique, influencé de manière 
significative dans la pratique par la réflexivité des dirigeants et des agents publics.

\subsection{La réflexivité des acteurs : un nouveau levier de gestion ?}

Dans leur approche rationnelle, les gestionnaires ont souvent proposé des instruments relativement simples pour les aider à orienter les décisions et les actions dans la « bonne » direction. Les outils de gestion, compte tenu de leurs postulats fondateurs « simplistes », se sont davantage technicisés qu'adaptés à la complexité des situations de gestion, au risque de se voir dévalorisés.

Simons (1995), dans son modèle des leviers de contrôle (figure 1), revient sur ce paradoxe entre complexité des situations et simplifications des outils de gestion en opérant une double distinction entre, d'une part, des systèmes de contrôle «focalisés sur l'attention » opposés à des systèmes tournés vers la recherche d'opportunités et l'apprentissage et, d'autre part, des systèmes chargés d'encadrer les domaines stratégiques opposés à des systèmes pour formuler et mettre en œuvre la stratégie.

\section{Figure 1 : les niveaux de contrôle}

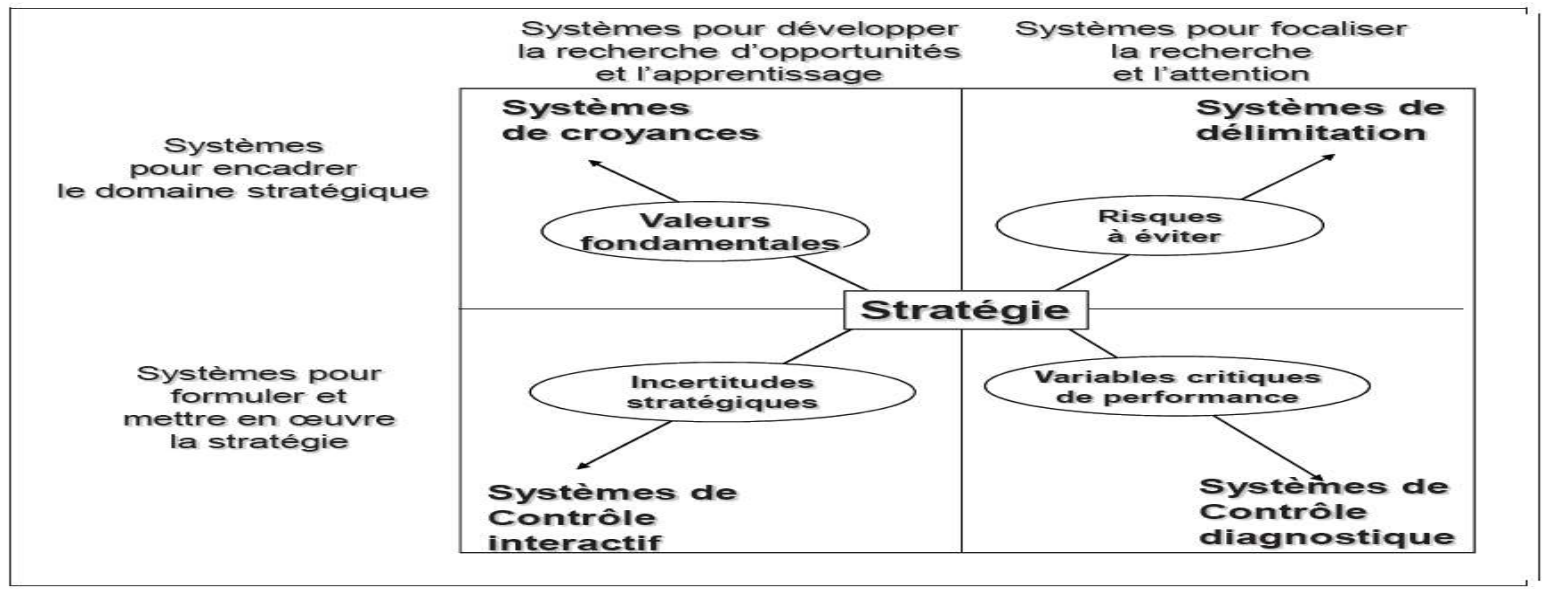

\section{R. Simons (1995)}

Dans ce modèle, au-delà des systèmes de croyances et de limites, c'est plus particulièrement entre contrôle diagnostique et contrôle interactif, que se joue l'opposition entre deux paradigmes de contrôle et deux formes de rationalité : le modèle de la régulation cybernétique assise sur la rationalité substantive et celui de l'apprentissage organisationnel (Lorino 1995 ; Mevellec 1990) et du management participatif (Drucker 1954) inspiré par la rationalité procédurale (Simon 1947). Afin de contourner quelque peu cette opposition, nous suggérons que la réflexivité peut représenter un levier de contrôle interactif en mesure de décrire les interactions entre acteurs et structure. Son étude peut permettre de décrire les mécanismes d'appropriation des outils d'évaluation de la performance en se positionnant sur l'analyse des simplifications définies par les acteurs dans l'utilisation de ces instruments. 
Pour aborder cette question, nous utilisons le modèle de la réflexivité définie par l'approche « réaliste critique » d'Archer, (2003, 1995, 1982) car il permet d'appréhender les représentations des acteurs dans leur mode réflexif. A partir du modèle de transformation adaptative de Bhaskar (1989), la sociologue propose de modéliser tout processus social en trois temps récursifs :

1- L'explication du contexte de conditionnement qui vise à mettre en lumière les structures qui vont contraindre ou faciliter l'action;

2- L'explicitation de l'action qui va être élaborée puis mise en œuvre par différentes catégories d'agents;

3- L'analyse du résultat de l'action sociale, qui peut reproduire ou transformer le contexte initial de conditionnement.

Les simplifications de la complexité par les usages des instruments d'évaluation de la performance peuvent s'appréhender à partir d'une analyse récursive de ce genre. La construction de ces simplifications ne se réalise jamais en « vase clos» au contraire elle est le fruit d'un processus réitératif qui est très proche de la réflexivité des « réalistes critiques ». Suivant la pensée d'Archer, cette situation montre que « les actions sont plus fortes que le pouvoir conditionnant des structures et que les usages transforment le système des rôles en place » (2003). Dans cette optique, l'artefact rationaliste fait place à la conception de l'usage « réflexiviste » au centre du cycle d'appropriation des outils de gestion. La performance dépend du degré d'interprétation et d'apprentissage par ses acteurs. Son obtention est le résultat de la résolution, au fil de l'eau, de nombreuses situations paradoxales entre actions convergentes de nombreux acteurs de l'organisation (concepteurs, codéveloppeurs, etc.) et non convergentes de parties prenantes du projet (pouvoirs publics, organisations non gouvernementales, etc.).

Archer met la réflexivité au centre de ce processus de résolution des paradoxes entre les structures et les actions. Elle élabore une critique à l'endroit des approches holistes, individualistes et de réciprocité et elle cherche à réhabiliter le « dualisme analytique » pour reconnaître l'indépendance de l'agent envers la structure et vice versa.

Cette posture «réaliste critique » nous pousse à croire que c'est dans la réflexivité et pas uniquement dans la rationalité (même limitée) des agents que se trouvent les principales explications des simplifications de la complexité opérées à l’Université. Giddens (1984) parle d'ailleurs de « réflexive monitoring » (pilotage réflexif) pour décrire « ce retour continu qu'effectue un individu sur ses actes et à partir de ses actes » (de Vaujany, 2006) afin de surveiller et de contrôler ses modes de réflexion et d'action. Néanmoins, Archer s'oppose à la dualité de la théorie 
de la structuration (Giddens, 1984) et elle privilégie le «dualisme analytique » qui distingue structure et action (agency) bien qu'elles se combinent dans la vie sociale. En distinguant, dans l'analyse, structure et action, elle favorise la lecture de la réflexivité des acteurs dans sa phase « active ».

Archer (1995) reprend le concept de morphogenèse (figure 2) utilisée notamment en biologie (végétale ou animale) pour décrire les changements dans la forme de la relation entre action et structure. Pour Archer, « les systèmes sociaux émergent des actions et interactions entre agents ». Cette émergence est rendue possible grâce à la notion de temps, élément essentiel de la théorie d'Archer. Les actions et interactions des agents s'inscrivent forcément dans un contexte temporel spécifique où des systèmes structurels préexistent aux actions et aux interactions. Leurs actions et interactions vont être conditionnées par ces structures sans pour autant être totalement déterminées. Ils peuvent soit décider simplement de les reproduire (c'est l'homéostasie ») ou de les changer (Archer parle de morphogenèse). Les relations entre structure et action sont donc à la fois temporelles et cycliques.

Figure 2 : La morphogenèse des systèmes et l' " agency "

$\mathrm{T} 1$ $\mathrm{T} 2$ $\mathrm{T} 3$ $\mathrm{T} 4$

\begin{tabular}{|c|c|c|}
\hline $\begin{array}{c}\text { Propriétés structurelles } \\
\text { (conditions matérielles) }\end{array}$ & \\
Propriétés culturelles & Actions et interactions & Émergence des propriétés pour \\
(valeurs, croyances) & le prochain cycle \\
Propriétés agentielles & & \\
(dispositions naturelles) & & \\
& & \\
\hline Agent primaire conditionné & Agent de corps en interaction & Identité personnelle et sociale \\
\hline
\end{tabular}

D’après M-S. Archer (1995 et 2002)

Le «dualisme analytique » se fonde sur deux propositions très simples : la structure est nécessairement antérieure aux actions qui la transforment; l'élaboration de la structure est nécessairement postérieure à ces actions. Les relations entre structure et action font apparaitre une double causalité et un double conditionnement. Les actions passées des individus contraignent ou permettent les actions et interactions présentes. L'utilisation du temps dans l'analyse de la relation «agency - structure » permet d'éviter le mélange entre les deux. L'étude des simplifications de la 
complexité par les usages des indicateurs peut tirer parti de ce «dualisme analytique » en approchant la réflexivité des acteurs universitaires à l'intersection de la phase interactive (T2-T3) de la morphogenèse décrite par Archer.

Pour faciliter l'application du dualisme analytique, Archer distingue deux sortes d'agents (figure 2). Les "agents primaires" associés à chaque individu faisant partie d'une collectivité ayant des intérêts et des objectifs qui lui sont propres. Ensuite, à partir de leurs interactions, ces agents primaires se transforment collectivement en «agents de corps » lorsqu'ils tentent activement de changer leur environnement social. Les agents sont à la fois constitués par le social et constituent le social (Archer, 2002, p.11). Ils sont des êtres réflexifs et l'on ne sait jamais comment ils vont changer leur environnement social. Par contre, on peut essayer de comprendre comment se construit leur réflexivité dans les interactions avec leur environnement (naturel, pratique et social). Les agents possèdent une identité personnelle et collective produite à partir de leurs interactions avec leur environnement (ibid., p.15). A partir de là, ils acquièrent également une identité sociale (T3-T4) suivant le rôle qu'ils choisissent de jouer.

Nous faisons l'hypothèse que les simplifications de la complexité par les usages représentent des instruments de réflexivité utilisés par les agents pour construire leur identité à partir de leurs interactions sociales. Pour cela, nous approchons ces simplifications au cours de l'étape T2-T3 d'interactivité du cycle morphogénétique d'Archer. L'auteure perçoit l'interaction sociale comme conditionnée par la structure, mais jamais déterminée par elle puisque les agents possèdent « leurs propres pouvoirs émergents irréductibles » (1998). Les nouvelles propriétés de la structure qui apparaissent par la suite sont considérées comme des conséquences largement non intentionnelles. La modification des propriétés structurelles antérieures et l'introduction de nouvelles propriétés sont le produit réflexif des différents résultats que recherchent simultanément les diverses parties prenantes. Par la suite, cette élaboration structurale fait démarrer un nouveau cycle morphogénétique puisqu'elle fait entrer en jeu de nouveaux facteurs qui influent sur l'interaction, soit en la contraignant soit en la facilitant. T4 devient ainsi le nouveau T1.

L'activité de l'individu peut être autonome, mais elle est très significativement déterminée. L'individu est le processus par lequel «un acteur essaie de se constituer comme un sujet en empruntant aux divers registres de l'action dans lequel il est enserré » (Archer 2003). La réflexivité devient indispensable à la définition de tout individu puisqu'il ne peut exister de « réflexivité possible chez un acteur enfermé dans une seule logique d'action » (ibid.).

Dans cette perspective, le «dualisme analytique » proposé par le «réalisme critique »d'Archer permet, dans la pratique, d'intégrer les préoccupations de l'individualisme méthodologique et de 
l'holisme (Dubet, 2002) par une étude, dans le temps, des interactions récursives entre structure et action. Archer reconnaît l'articulation du rapport entre l'individu et la société en sociologie comme une variation de la problématique de la structure et de l'action (au même titre que les oppositions classiques entre le volontarisme et le déterminisme ou encore le subjectivisme et l'objectivisme). Ceci lui permet de dépasser la « réciprocité » structure/action du relationnisme de Giddens (1984) ou du constructivisme social de Berger et Luckmann (1986) en approchant la réflexivité de manière diachronique. Les structures dépendent de «certaines actions posées à certains moments précis » qui facilitent l'étude des usages des instruments par les acteurs en isolant la réflexivité propre à chaque action. C'est cette posture méthodologique que nous privilégions ici car elle nous permet d'approcher la problématique de l'appropriation par simplification par les usages des indicateurs de la performance à travers les interactions entre instruments et agents.

\section{Appropriation des instruments d'évaluation et niveaux de contrôle public}

\subsection{Cadre conceptuel}

Selon certaines conceptions, les qualités intrinsèques des outils suffisent à les définir, voire à expliquer leurs « réussites ». Si ces qualités sont suffisamment pertinentes en termes de gain d'efficacité et d'efficience pour l'organisation qui les reçoit, la rationalité substantive des acteurs devra favoriser quasi-mécaniquement son usage, suivant le principe selon lequel « l'intendance suivra » (Grimand 2006). Mais cette conception relève essentiellement du modèle de la rationalité instrumentale (Hatchuel et Weil 1992); les décisions sont considérées comme rationnelles, les acteurs se conforment aux règles proposées, l'information est parfaite et les capacités cognitives des acteurs sont infinies. Dans cette perspective, l'outil de gestion est perçu comme l'instrument, par lequel la performance de l'organisation se verra augmentée.

De Vaujany (2005) oppose à ces modèles rationalistes des modèles réflexifs qui s'intéressent aux capacités réflexives des acteurs. Le gestionnaire est un agent réflexif qui cherche essentiellement à maintenir un équilibre dans ses schèmes socio-cognitifs (plutôt qu'à augmenter sa rationalité). L'organisation comme les outils de gestion sont des propriétés du structurel qui peuvent habiliter ou contraindre l'action; le changement structurel est un processus récursif et interactif (de Vaujany parle de processus psychosociologique).

Les travaux portant sur l'appropriation des outils de gestion (Grimand 2006 ; de Vaujany 2005) insistent sur la nécessité de prendre en compte toute la complexité des situations d'interprétation des acteurs lors de la mise en œuvre d'un outil. De Vaujany soutient qu'il ne suffit plus de s'intéresser à «l'adoption» des outils (en tant que processus de décision) mais à leur «appropriation » pour 
notamment mesurer les écarts constatés entre l'usage prévu d'un outil et, son utilisation effective. L'usage devient central dans la démarche de compréhension de l'appropriation des outils par les acteurs car l'usage prévu va se trouver rejeté, modifié et simplifié ou approuvé par l'utilisateur.

La théorie de "l'appropriation » met en avant les interactions entre des acteurs et des outils pour expliquer la construction de normes de gestion. Cette approche intègre le « caractère irréaliste des hypothèses de rationalité intégrées dans les outils par rapport aux systèmes de rationalités locales en interaction que constituent les organisations » (Moisdon 1997). C'est autour de ces insuffisances des outils de gestion que les acteurs vont construire des usages parfois conformes, parfois déviants, le plus souvent simplifiés, notamment dans les organisations complexes. Conception et usage des outils de gestion s'intègrent dans un vaste processus récursif et continu. Après sa phase d'étude et de conception, l'outil est approprié par les acteurs qui l'interprètent, l'adaptent, le déforment ou le reforment à partir de leurs propres représentations de la performance. Puis ces mêmes acteurs ou d'autres se réapproprient l'outil reconstruit à partir de son usage. «Les savoirs, en partie théoriques, détenus par les prescripteurs à l'origine du processus, ne s'éprouvent qu'au contact des destinataires de l'expertise; c'est au cours de ces échanges réciproques qu'ils se «contextualisent » et prennent un sens concret pour les opérationnels » (Aggeri et Hatchuel 1997, p. 244). Cette approche rejoint celle des «réalistes critiques» portant sur la réflexivité des acteurs en situation d'actions et d'interactions (Archer et al.1998; Bhaskar 1989).

Pour ces deux cadres théoriques, les usages des instruments de gestion peuvent être rapprochés, dans le but de considérer l'outil comme un artefact inscrit dans un processus de construction. En effet, ils empruntent à la théorie de « la structuration » (Giddens 1984) et à celle de «l'enactment » (Weick 1995) l'approche duale structure/action mais attribuent aux interactions une dimension récursive tant dans l'usage que dans la réflexion relative à l'outil. Ces deux perspectives théoriques ont pour point commun d'insister sur le rôle actif de l'utilisateur face à l'outil et d'introduire l'intervention de la réflexivité à côté des rationalités substantive ou limitée. L'outil de gestion retrouve une certaine extériorité. Il est, dans la vision d'Archer, «un système d'action correspondant à un niveau d'interaction qui favorise l'appropriation par l'usage » (de Vaujany et Grimand 2005) et sa reconnaissance va dans le sens d'un contrôle interactif de la performance.

Le modèle que nous appliquons ici, est tiré de l'analyse réaliste critique d'Archer. Il porte sur « un outil en usage » permettant de faire le lien entre réflexivité et appropriation et de saisir toute la complexité des situations qui mène à l'usage des outils de gestion. En effet, l'artefact est l'élément « objectif », visible du nouvel outil (Lorino 2002). C'est sur cet élément que vont se constituer les attitudes envers l'artefact. Par contre, dans la pratique, les utilisateurs vont investir cet artefact de 
nouveaux usages plus complexes en formalisant des simplifications afin de rapprocher la multiplicité des instruments de gestion et la diversité des rationalités en jeu à l'Université.

Ici, nous nous focalisons sur l'étape de simplification de la complexité par les usages car elle s'élabore à partir d'une «contextualisation interactive» des simplifications des représentations construites précédemment (figure 3).

Nous proposons un cadre conceptuel (figure 3) qui, dans un premier temps, rapproche les simplifications et les formes de rationalité reconnues en gestion puis qui met en parallèle le processus d'appropriation des outils de gestion et celui de contrôle de gestion pour affirmer leur proximité et l'intérêt de les analyser de manière symétrique.

Figure 3 : Simplifications de la performance et de ses outils de mesure

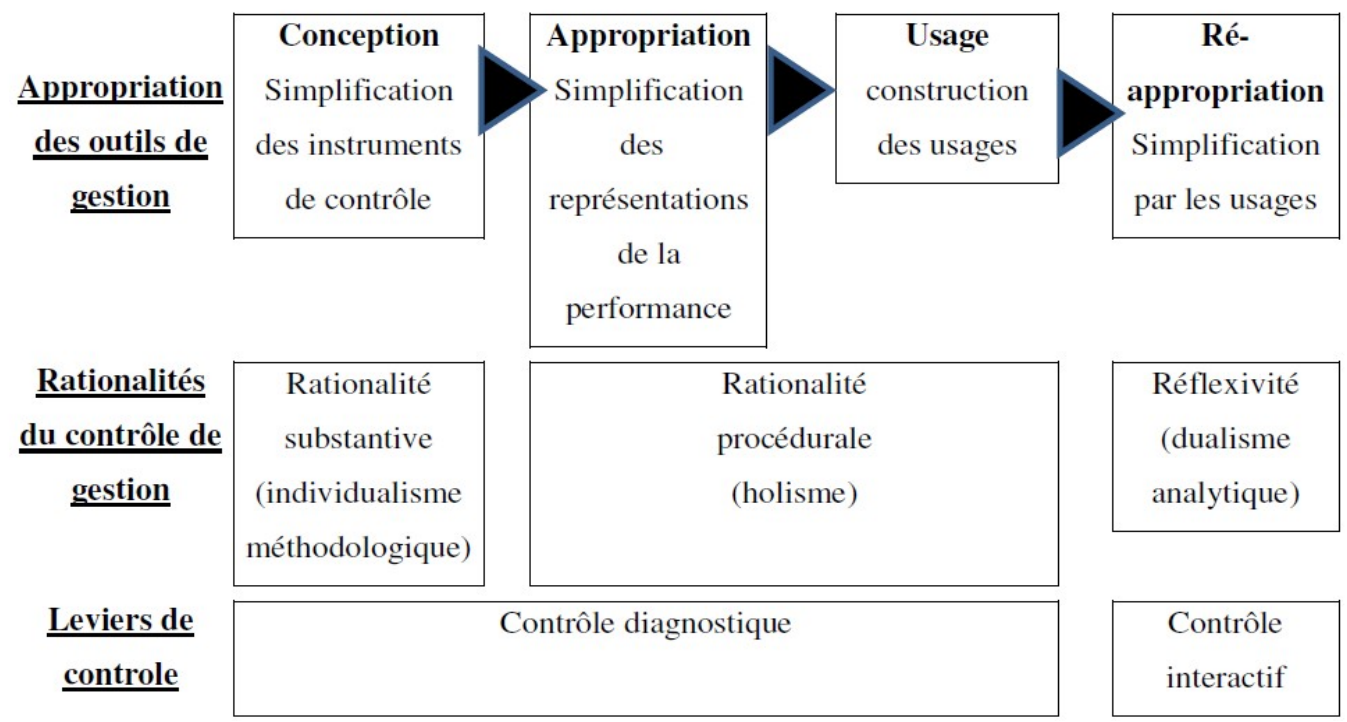

(Source : auteur)

Cette présentation, en faisant apparaître en parallèle à ce rapprochement, les leviers de contrôle mis en évidence par Simons (1995), interroge les sources de perfectionnement du management de la performance des universités françaises. L'éclairage sur la complexité des universités françaises qu'offrent les instruments d'évaluation de la performance se concrétise par une plus grande visibilité des simplifications et «digestions » (Chauvey 2010) de cette complexité. Pour servir ce projet de réflexion sur les formes d'appropriation des indicateurs de la performance universitaire, nous souhaitons effectuer un retour sur ces simplifications. Il ne s'agit pas de revenir sur le principe de la simplification des techniques mais d'en mettre en évidence l'hétérogénéité et l'utilité dans les processus d'appropriation des outils de gestion du NPM à l'Université.

L'hypocrisie des organisations (ici universitaires) mise en évidence par Brunsson (1989) opère un découplage entre les discours et les actions qui se concrétisent par, d'un côté, la mise en avant 
d'instruments d'évaluation rationnel et diagnostique, et d'un autre côté, l'existence de simplifications ou de déviances à ces discours (Mazouz et al., op .cit.). Au final, ces mécanismes soulèvent des questions essentielles assez peu étudiées. Comment se construit le processus d'appropriation des outils d'évaluation de la performance à l'Université ? Les simplifications de ses instruments d'évaluation sont elles des formes de cette appropriation ? Comment parviennent-elles à gérer les contradictions ou les paradoxes entre discours et actions ?

Selon nous, les individus sont obligés de développer, à côté des discours rationnels portés par les instruments de gestion du NPM, des solutions qui s'éloignent des consignes rationnelles afin d'éviter les dysfonctionnements que pourrait générer une absence de gestion des paradoxes. Les acteurs universitaires sont donc placés dans une situation d' "exploration » (March 2006) qui rend la manière dont ils simplifient leur réalité dépendante, d'une part, des paradoxes auxquels ils doivent faire face et, d'autre part, de leur position dans la gouvernance universitaire. Les résultats empiriques de notre étude, présentés par la suite, décrivent des formes multiples de simplifications dont l'analyse présente un intérêt manifeste dans la perspective de décrire les effets de l'appropriation des outils de gestion prescrits par le NPM.

\subsection{Méthodologie}

Notre étude des simplifications des représentations de la performance des universités et de ses instruments de contrôle a été réalisée à partir d'une analyse des indicateurs d'évaluation utilisés par l'A.E.R.E.S. (Agence d'Évaluation de la Recherche et de l'Enseignement Supérieur) et la D.G.E.S.I.P. (Direction Générale de l'Enseignement Supérieur et de l'Insertion Professionnelle) afin de mettre en évidence les rationalités appliquées dans la construction de leurs usages initiaux.

Pour étudier plus spécifiquement la simplification de la complexité par les usages, nous utilisons une enquête exploratoire menée auprès des présidents d'université de quatre-vingt-cinq universités françaises (taux de réponse de 48\%) et portant sur leurs représentations de la performance. Notre enquête a été réalisée à distance au cours de l'année universitaire 2011-2012 grâce à la collaboration de la Conférence des Présidents des Universités (CPU) qui l'a diffusée auprès des ses membres et qui, dans certains cas, en a assuré la réception.

La gouvernance actuelle des universités françaises accorde à leurs présidents, dans le mode d'élection et dans la pratique, de larges responsabilités qui nécessitent de leur part une vision étendue des objectifs et de la performance de leur université. Par ailleurs, la loi LRU a consacré l'élection des présidents d'université par le seul conseil d'administration (au lieu des trois conseils : Conseil d'administration, Conseil scientifique et Conseil de la vie étudiante dans le passé) en leur attribuant de nouvelles responsabilités (attribution de primes et droit de veto sur le recrutement des 
personnels) assez peu démenties, en 2013, par la loi sur l'Enseignement Supérieur (dite loi « Fioraso »). Ce contexte attribue aux présidents des universités françaises une place prépondérante dans le pilotage de la performance de leur structure mais aussi une confrontation plus nette encore aux contraintes supra et infra organisationnelle de leur gouvernance.

Initialement, cette enquête portait sur un ensemble de trente questions relatives au pilotage de la performance et à ses instruments d'évaluation et de contrôle. Nous retenons de cette enquête les réponses aux six questions suivantes portant sur les perceptions de la performance et sur l'usage des indicateurs:

1- Dans quels domaines jugez-vous votre université non ou sous performante?

2- Citez les activités pour ou dans lesquelles votre université vous semble performante?

3- Parmi les indicateurs suivants, lesquels permettent, d'après vous, de mesurer le mieux la performance de votre université ?

4- Citez les indicateurs qui vous semblent les plus représentatifs de la performance d'une université ?

5-Pour votre université, vous parait-il nécessaire de développer des indicateurs de performance spécifiques? Si oui, lesquels?

6- Quels sont les indicateurs les plus utilisés dans le pilotage de la performance de votre université ? Chacune de ces questions et les réponses proposées ont été construites après analyse des indicateurs les plus utilisés par l'A.E.R.E.S. et la D.G.E.S.I.P. afin de mesurer leurs effets sur les représentations et les actions des présidents.

Les réponses fermées ont, pour la plupart, fait l'objet d'un croisement entre plusieurs questions afin de mesurer la place des indicateurs prescrits dans les usages universitaires. La question 3 reprend un ensemble d'indicateurs prescrits par l'A.E.R.E.S. et la D.G.E.S.I.P. dans leurs évaluations associés à des indicateurs construits dans une majorité des tableaux de bord prospectifs des universités que nous avons étudiés. Le croisement des réponses obtenues à cette question avec celles obtenues à la question 1 et 2 permettent de déceler un certains nombre de paradoxes de l'évaluation de la performance universitaire qui nécessitent une simplification des indicateurs dans leurs perceptions et dans leurs usages. La question 4 propose aux répondants de décrire de manière plus informelle les indicateurs les plus représentatifs de la performance universitaire afin de mesurer les niveaux de simplifications à l'usage. Enfin les questions 5 et 6 plus ouvertes sont destinées à faire apparaître à la fois les indicateurs en usage dans les tableaux de bord des universités ainsi que les domaines dans lesquels les évaluations prescrites semblent insuffisantes. L'utilisation de réponses fermées et 
ouvertes dans les différentes questions formulées a permis, lors de l'analyse de l'enquête, de mettre en évidence les écarts entre les indicateurs prescrits et les usages tout en mesurant les simplifications subjectives et objectives opérées par la réflexivité des directions universitaires.

Les questions fermées (question 1 à 3) portaient sur les niveaux de performance perçus par les répondants et les indicateurs en mesure de les évaluer. Elles ont été formulées à partir d'une échelle de Likert à cinq niveaux ( $1=$ pas du tout d'accord ; $5=$ tout à fait d'accord). Les réponses ont été analysées par un logiciel d'analyse statistique (SPSS Version 20.0) afin de réaliser une analyse en composante principale et de décrire les principaux indicateurs de la performance identifiés par les présidents. Les réponses aux questions ouvertes (question 4 à 6) ont été analysées sur SPSS à "partir d'une catégorisation a posteriori des données afin de procéder à des regroupements d'indicateurs. Ce type d'étude peut faire l'objet de certains biais d'interprétation mais, dans le cas de cette analyse, l'encodage des données ouvertes a révélé une importante proximité des indicateurs ou des niveaux de performance identifiés par les présidents. Ceci nous permet de présenter des résultats synthétiques (tableau 3) faisant apparaître des indicateurs soit, cités par les présidents d'université (tableau 1), soit, explicitement révélés par l'encodage que nous avons réalisés (tableau 2).

L'étude de la réflexivité des présidents d'université a été menée à partir de cette double analyse de réponses fermées et ouvertes qui restitue à la fois les perceptions de la performance des présidents d'université et qui permet également de connaître les indicateurs de la performance à l'usage dans les universités. Pour Archer (1995), les acteurs construisent leur réflexivité soit à partir de d'interprétations représentées ici par les réponses à nos questions fermées, soit en empruntant à des registres d'action décrits par les réponses à nos questions ouvertes.

Plus globalement, par cette approche méthodologique, nous cherchons à analyser les trois temps réflexifs mis en évidence par Archer (2003). L'explication de la structure et du contexte de l'évaluation de la performance universitaire est menée à partir de notre analyse des indicateurs prescrits par l'A.E.R.E.S. et la D.G.E.S.I.P. L'analyse de l'agent en interactions est fournie par l'étude des perceptions de la performance des présidents d'université (question 1 à 3). L'explication du résultat de la transformation de la structure est établie à partir de l'analyse des usages des indicateurs de la performance formulés dans les réponses aux questions 4 à 6 .

\section{Résultats et discussions}

La première forme d'appropriation que révèle notre étude empirique est définie par les simplifications opérées dans la construction des indicateurs de performance prescrits par 
l'A.E.R.E.S. ou la D.G.E.S.I.P. Ces deux structures reprennent les indicateurs intégrées dans les programmes ministériels 150 (Formations supérieures et recherche universitaire), et 231 (Vie étudiante) et les regroupent par thème (Performance scientifique, lisibilité et visibilité des formations en leur attribuant des valeurs cibles souvent communes à tous les établissements évalués). Nous recensons (tableau 3) les principaux thèmes évalués et les indicateurs majeurs par thème afin de décrire cette forme de simplification construite à partir de la valeur-caution que lui donne son origine ministérielle.

A partir de notre enquête menée auprès des présidents d'université et des questions portant sur leurs perceptions de la performance, nous voyons apparaître une deuxième de forme d'appropriation par simplification à rapprocher de la valeur-assimilation proposée par de Vaujany (ibid.). Le tableau 1 recense la fréquence des citations des indicateurs de performance jugés par les présidents d'université comme représentatifs de la performance (question 2 et 3) ou de la sous performance (question 1) de leurs universités. Il retient, à partir d'une analyse en composante principale, les indicateurs les plus cités par les présidents d'université pour juger de la performance de leur établissement. Ainsi, lorsqu'on les interroge sur les indicateurs de performance qu'ils jugent significatifs, les présidents des universités réduisent très sensiblement le nombre de thème d'évaluation et d'indicateurs qu'ils jugent importants. Cette assimilation s'opère à la fois par une reprise des indicateurs prescrits (taux d'insertion professionnelle), par une réinterprétation de ces indicateurs (taux d'échec des étudiants plutôt que de taux de réussite en Licence, nombre de publications plutôt que nombre d'enseignants publiants) ou par une description de nouveaux indicateurs non prescrits (taux d'encadrement des étudiants, nombre d'étudiants étrangers, partenariats Université-entreprises).

Tableau 1. Principaux indicateurs représentatifs de la performance universitaire

\begin{tabular}{|c|c|}
\hline Indicateurs & Pourcentage \\
\hline Le taux d'insertion professionnelle des étudiants & $14,4 \%$ \\
\hline Indicateurs de suivi des anciens étudiants & $12,1 \%$ \\
\hline Taux d'échec des étudiants & $11,4 \%$ \\
\hline Taux d'encadrement des étudiants & $11,1 \%$ \\
\hline Nombre de publications scientifiques & $10,4 \%$ \\
\hline Partenariats Université-entreprises & $9,8 \%$ \\
\hline Nombre d'étudiants étrangers & $9,4 \%$ \\
\hline Part des diplômes en formation continue & $4,8 \%$ \\
\hline Taux d'occupation des locaux & $4,4 \%$ \\
\hline Respect des contraintes budgétaires & $3,7 \%$ \\
\hline Respect des normes de performance nationale ou internationale & $3,1 \%$ \\
\hline Nombre d'étudiants & $2,7 \%$ \\
\hline Nombre de formations & $1,7 \%$ \\
\hline Autres & $1,0 \%$ \\
\hline Total & $100,0 \%$ \\
\hline
\end{tabular}


Cette appropriation s'exécute par réduction et simplification du nombre d'indicateurs afin d'adapter les critères d'évaluation au contexte particulier de ces universités et de réduire le risque de tensions de gouvernance publique (Mazouz et al, 2012). Elle génère aux yeux des présidents d'université une simplification managériale (figure 4) qui leur permet d'adapter leurs objectifs et d'optimiser leur démarche stratégique. Cette assimilation est souvent perçue par les présidents d'université à la fois comme une réponse aux impératifs d'évaluation prescrits par leur tutelle mais aussi comme un perfectionnement de leur outillage d'évaluation et de leur pilotage de la performance.

La simplification à l'usage que nous analysons par la suite est issue des réponses des présidents d'université aux questions portant sur les indicateurs les plus utilisés et qu'ils jugent déterminants dans leur gouvernance (tableau 2). Nos analyses concluent à une simplification très conséquente qui réduit l'évaluation à quatre indicateurs majeurs portant soit sur la gestion des moyens (évolution de la masse salariale, nombre d'étudiants présents aux examens), soit sur la visibilité de leur université (taux d'insertion professionnelle, taux d'échec des étudiants). La volonté des dirigeants universitaires de rendre leur structure visible et bien dotée s'accompagne d'un usage simplifié des indicateurs de la performance soit, par réappropriation d'indicateurs prescrits (taux d'insertion professionnelle) ou assimilés (taux d'échec des étudiants), soit, par réinterprétation d'impératifs financiers suggérés par les dotations ministérielles (nombre d'étudiants prescrits aux examens ou évolution de la masse salariale).

Tableau 2. Catégorisation de l'usage des indicateurs de la performance universitaire

\begin{tabular}{|l|c|}
\hline \multicolumn{1}{|c|}{ Indicateurs } & Pourcentage \\
\hline Le taux d'insertion professionnelle des étudiants & $\mathbf{1 7 , 4 \%}$ \\
Nombre d'étudiants présents aux examens & $\mathbf{1 6 , 4 \%}$ \\
Taux d'évolution de la masse salariale & $\mathbf{1 4 , 1 \%}$ \\
Taux d'échec des étudiants & $\mathbf{1 2 , 2 \%}$ \\
Nombre de publications scientifiques & $\mathbf{8 , 4 \%}$ \\
Respect des contraintes budgétaires & $\mathbf{7 , 1 \%}$ \\
Taux d'encadrement des étudiants & $\mathbf{6 , 7 \%}$ \\
Taux d'occupation des locaux & $\mathbf{6 , 4 \%}$ \\
Nombre d'étudiants & $\mathbf{5 , 0 \%}$ \\
Nombre de formations & $\mathbf{5 , 0 \%}$ \\
Autres & $\mathbf{1 , 3 \%}$ \\
\hline \multicolumn{2}{|c|}{ Total } \\
\hline
\end{tabular}

Nous parlons de simplifications opérationnelles (figure 4) pour décrire cet usage d'indicateurs majeurs car elles se justifient par des exigences opérationnelles quelles soient dictées par le mode d'attribution des moyens des universités françaises (masse salariale ou étudiants présents aux examens) ou par l'appropriation par assimilation des indicateurs prescrits. Cette simplification à l'usage se concentre sur des indicateurs synthétiques qui présentent l'avantage pour les présidents d'université de répondre à des exigences budgétaires et d'attractivité de leurs universités (ouverture 
et visibilité des formations). Par contre, elle réduit très significativement l'évaluation de la performance à quelques missions (visibilité des formations, accompagnement des étudiants, relations extérieures) tout en négligeant d'autres perçues, au niveau sociétal ou politique, comme essentielles (performance scientifique, autoévaluation des universités, gestion des infrastructures).

Le tableau 3 propose une confrontation des résultats des analyses des indicateurs prescrits avec les perceptions (tableau 1) et les usages (tableau 2) des indicateurs d'évaluation de la performance. Il met en évidence les trois formes d'appropriation par simplification que nous avons pu observer et que l'on peut rapprocher des modes d'appropriation caution-assimilation-usage décrits par de Vaujany (2005).

Tableau 3. L'appropriation par simplification des indicateurs de performance universitaire

\begin{tabular}{|c|c|c|}
\hline $\begin{array}{c}\text { Domaines d'évaluation de la } \\
\text { performance (caution) }\end{array}$ & $\begin{array}{c}\text { Appropriation des indicateurs } \\
\text { de la performance (assimilation) }\end{array}$ & $\begin{array}{c}\text { Simplifications à l'usage } \\
\text { (usage) }\end{array}$ \\
\hline $\begin{array}{l}\text { - Performance scientifique } \\
\text { (nombres d'enseignants publiants) } \\
\text { - Lisibilité et visibilité des } \\
\text { formations (nombre de primo- } \\
\text { entrants, taux de réussite en L3, } \\
\text { taux d'insertion professionnelle) } \\
\text { - Qualité de l'accompagnement et } \\
\text { de la vie étudiante (nombres } \\
\text { d'associations étudiantes, nombre } \\
\text { d'étudiants élus) } \\
\text { - Relations extérieures } \\
\text { (internationales, } \\
\text { interuniversitaires et universités- } \\
\text { entreprises) } \\
\text { - Gouvernance et auto-évaluation } \\
\text { des universités (nombre de } \\
\text { démarches d'autoévaluation et de } \\
\text { certification qualité) } \\
\text { - Gestion des infrastructures (taux } \\
\text { d'occupation des locaux, } \\
\text { pourcentage de personnels } \\
\text { techniques et administratifs) }\end{array}$ & $\begin{array}{l}\text { - Réussite des étudiants (taux d'échec } \\
\text { des étudiants, représentation des } \\
\text { étudiants dans la gouvernance) } \\
\text { - Spécificités scientifiques (nombre } \\
\text { de publications) } \\
\text { - Attractivité des formations (taux } \\
\text { d'insertion professionnelle et taux } \\
\text { d'encadrement des étudiants) } \\
\text { - Ouverture de l'université (nombre } \\
\text { d'étudiants étrangers, partenariats } \\
\text { entreprises) }\end{array}$ & $\begin{array}{l}\text { - Taux d'insertion professionnelle } \\
\text { (réussite, ouverture et visibilité des } \\
\text { formations) } \\
\text { - Taux d'échec des étudiants } \\
\text { (réussite et visibilité des formations) } \\
\text { - Nombre d'étudiants présents aux } \\
\text { examens (réussite et budget) } \\
\text { - Evolution de la masse salariale } \\
\text { (gestion budgétaire) }\end{array}$ \\
\hline
\end{tabular}


Un système social est toujours en quête d'un ordre simplifiant et orientant le foisonnement de la diversité des acteurs sans y parvenir totalement. Le paradoxe central à l'Université se situe bien dans cette incapacité à tout ordonner donc à rendre simple une organisation complexe. Amans (2003) ou encore Chatelain-Ponroy (2008) ont démontré dans leurs recherches respectives sur d'autres types d'organisations publiques (les organisations culturelles) que la simplification de la complexité par un nombre réduit d'indicateurs de performance (le nombre d'entrées dans un musée ou un théâtre par exemple) pouvait suffire à l'identification de l'organisation. Nous pensons également que la construction identitaire ne peut se réaliser dans les organisations universitaires qu'à partir d'une simplification significative de la complexité du concept de performance qui rapproche la profusion des rationalités et la diversité des acteurs.

Nous avons remarqué, dans nos analyses de terrain, que cette simplification peut prendre des formes diverses qui impactent de manière réflexive les propriétés institutionnelles, structurelles et agentielles qu'Archer (2003) attribuent à tout système social. La mesure de ces impacts est, à notre sens, à rapprocher de celle que réalisent Mazouz et al. (2012) par la description de tensions institutionnelles, organisationnelles et managériales. Ceci nous amène à suggérer que les simplifications des indicateurs de la performance universitaire sont de trois ordres successifs (figure 4) auxquels il est possible d'associer à chacun un mode d'appropriation une forme de rationalité.

\section{Figure 4 : Les simplifications de la performance à l'Université}

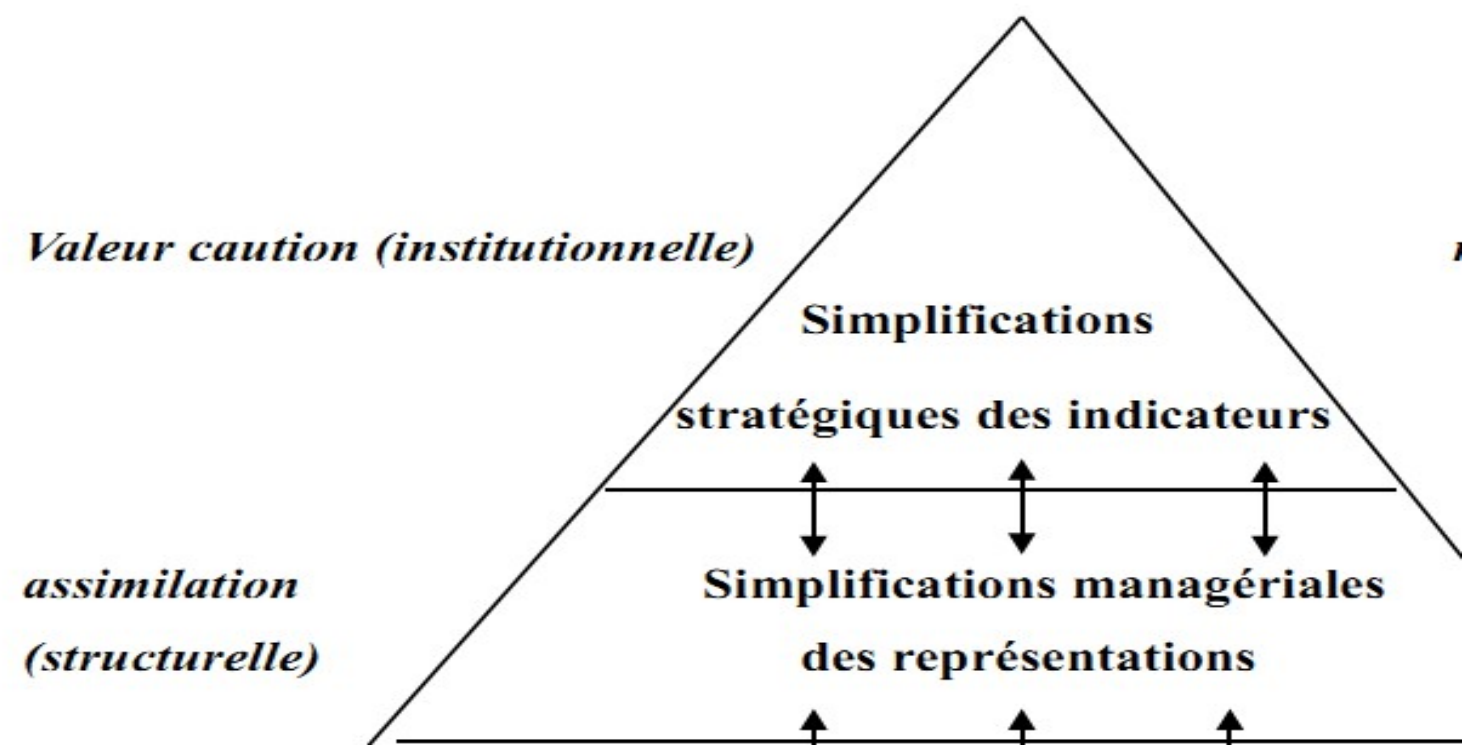

(Source : auteur)

Le premier ordre est prescrit par l'autorité administrative de tutelle qui par la promotion de systèmes homogènes d'évaluation, décrit un premier type de simplifications stratégiques. Il 
correspond plutôt à l'exercice d'une rationalité substantive dont la finalité est d'attribuer une légitimité sociale aux objets et indicateurs d'évaluation (de Vaujany, 2007).

Le second ordre de simplifications est défini par les présidents d'université qui, par leur connaissance du contexte universitaire et des enjeux de pouvoir, simplifient les objectifs de performance en vue d'un management efficace et d'une gestion des conflits. Il s'agit d'une étape d'assimilation par l'organisation qui se réalise sous l'impulsion des managers afin de faire accepter la stratégie par les acteurs. La rationalité mise en œuvre est ici beaucoup plus procédurale.

La simplification opérationnelle par les usages est le troisième ordre de simplification qui est construit par les réinterprétations et les adaptations en fonction de paramètres locaux (ibid.). La réflexivité des acteurs universitaires est à ce niveau un élément essentiel de la simplification car elle guide le choix des indicateurs utilisés en réduisant leur nombre et en hiérarchisant les missions universitaires initialement prescrites.

Le second ordre de simplification correspond potentiellement à un niveau de performance conjointe (Reynaud, 2003) qui fait la synthèse entre les performances officielle et autonome associées respectivement aux simplifications stratégiques et opérationnelles. En effet, les bureaucraties professionnelles se caractérisent par une performance professionnelle et autonome difficilement contrôlable mais qui représente une grande part de la performance globale. Ne pas identifier cette performance dans les instruments d'évaluation de la performance revient à la faire disparaître progressivement et à remettre en cause une part significative de la performance globale de l'organisation. Dans les organisations fortement hiérarchisées, les actions non contrôlées ou non évaluées sont progressivement négligées par les acteurs qui, par anticipation rationnelle, se concentrent sur celles qui sont contrôlées. Les trois formes de simplification que nous identifions dans ce travail permettent de contrôler les performances officielle et autonome en évaluant une performance conjointe (ibid.). Les simplifications de la complexité par les usages des outils d'évaluation de la performance sont une expression de cette performance conjointe car elles sont, dans un premier temps, principalement réalisées par les présidents d'université dont nous avons essayé d'analyser la réflexivité face à ces outils.

\section{Conclusion}

Nous avons testé l'efficacité d'une analyse de la réflexivité des acteurs dans le cadre d'une large réflexion sur les processus d'appropriation par simplification de l'usage des indicateurs de la performance universitaire. Ce travail est une première description de l'instrumentation qui s'opère dans les universités à partir de la construction d' « habiletés » et d'usages (Marquet et Leroy, 2004) 
exprimée par la mise en commun d'instruments de gestion à la fois anciens (prescrits) et nouveaux (créés). A notre connaissance, les débats portant sur l'introduction des préceptes du NPM dans le management des universités se sont jusqu'alors surtout concentrés sur son instrumentalisation à travers la professionnalisation des agents publics et des outils qu'ils manipulent. Or, l'introduction d'un instrument de gestion dans une activité ne garantit rien sur son usage. Le processus « artéfactuel » qui se développe par la suite est très riche d'informations portant sur ses formes d'appropriation (instrumentalisation) mais aussi sur son utilisation par les acteurs (instrumentation). Nous avons proposé une tentative d'analyse de ce processus à partir de la réflexivité produite par les présidents d'université à travers leurs représentations et leurs usages des indicateurs d'évaluation. L'analyse des formes d'appropriation des indicateurs d'évaluation à partir de la réflexivité des acteurs constitue à notre sens une contribution originale à la description pratique de l'instrumentalisation et de l'instrumentation de ces outils. En effet, l'indicateur d'évaluation de la performance en tant qu'artefact recèle un grand potentiel descriptif en mesure de mieux comprendre les réinventions et détournements d'usage. La conception d'un outil de gestion ne débutant pas toujours là où on le croit et n'étant jamais réellement terminée (Lorino, 2002).

Notre recherche contribue à décrire le rôle des acteurs universitaires (et plus particulièrement celui des présidents d'université) face aux indicateurs d'évaluation de la performance. A la suite notamment de la théorie instrumentale (Rabardel, 2005) et de «l'approche théorique par les instruments » (Aggeri et Labatut, 2010), nous introduisons la réflexivité comme une autre forme de rationalité pratique, en mesure de mieux cerner le rôle des acteurs dans l'appropriation et l'usage des instruments de gestion issus notamment du NPM. Nous nous intéressons à cette réflexivité pour plusieurs raisons.

D'une part, en l'état actuel de la mise en œuvre des réformes universitaires (LRU notamment), nous considérons, que, par leur position de médiateur, les présidents d'université participent de manière prépondérante à la traduction des instruments officiels de gestion. Les simplifications qu'ils opèrent, déterminent en grande partie, parce qu'elles les anticipent, les simplifications opérationnelles représentées par les usages et bricolages locaux (Mazouz et al., 2012). Ceci les rapproche, par leur forme et leur contenu, des réflexivités mises en évidence par le courant « réaliste critique » mené par Archer.

D'autre part, les simplifications par l'usage des indicateurs de performance par les présidents d'université sont à la fois le produit de leurs représentations mais aussi une interprétation de celles des opérationnels. Il était donc important de se focaliser sur ces usages afin de tester l'efficacité d'une analyse de la réflexivité des acteurs en vue de décrire les formes d'appropriation des outils du 
NPM et de gestion des tensions qu'ils génèrent. Par contre, nous considérons que l'analyse des simplifications apportées par les autres acteurs universitaires (étudiants, enseignants, personnels administratif et technique) représente une perspective de recherche naturelle dont la densité et la temporalité nécessiteront sans doute la définition d'autres approches pratique et méthodologique.

Les simplifications opérées par les présidents d'université dans l'usage des indicateurs de performance s'inscrivent dans un processus plus large d'appropriation de ces outils. Elles peuvent être analysées comme une expression de la réflexivité « pratique » des acteurs qui permet d'enrichir la connaissance des processus de pilotage de la performance universitaire. Notre objectif était de mettre évidence les intérêts de l'étude de cette réflexivité en vue de mieux appréhender le processus d'appropriation des indicateurs de la performance universitaire. Il apparaît que, notamment par une étude des usages de ces indicateurs, il est possible de circonscrire certains éléments saillants de cette réflexivité.

Nous avons surtout souhaité mettre en avant les potentialités du «dualisme analytique » pour la description de cette réflexivité dans des situations d'évaluation de la performance publique. Les simplifications de la complexité par les usages des indicateurs ne sont qu'un type d'expression de la réflexivité des acteurs mais elles représentent une étape majeure du mécanisme d'appropriation des outils de gestion du NPM.

Il est important de replacer la réflexivité des acteurs au cœur du processus de construction de normes légitimes d'évaluation de la performance venant compléter l'appareillage classique prescrits par le NPM. Elle offre, en effet, une perspective de perfectionnement pratique voire de dépassement des postulats du NPM en définissant l'action réflexive comme un acte de gestion (subjectif) à part entière et en mesure d'accompagner la modernisation et la légitimation des instruments d'évaluation de la performance publique. 


\section{Références}

Aggeri, F., \& Hatchuel A. (1997). Les instruments de l'apprentissage. Construction et diffusion d'une expertise recyclage dans la conception automobile. In Moisdon, J.C. (éd.), du mode d'existence des outils de gestion (pp. 217-247). Paris : Séli Arslan.

Aggeri, F., \& Labatut, J. (2010). La gestion au prisme de ses instruments. Une approche généalogie des théories fondées sur les instruments de gestion. Finance Contrôle Stratégie (FCS), Septembre, 13(3), 5-37.

Amans, P. (2003). Les indicateurs de contrôle entre simplification et complexification - Le cas de la fréquentation muséale. Doctorat en Sciences de Gestion. Montpellier : Université de Montpellier II.

Archer, M.S. (1982). Morphogenesis versus structuration: on combining structure and action. The British Journal of Sociology, 22, 455-483.

Archer M.S. (1995). Realistic social theory: the morphogenetic approach. Cambridge : Cambridge University Press.

Archer M.S., Bhaskar R., Collier A., Lawson T. \& Norrie A. (1998), Critical realism, Essential readings, Routledge, Londres.

Archer, M.S. (1998). Théorie sociale et analyse de la société. Sociologie et sociétés, 30(1), 9-22.

Archer, M.S. (2002). Realism and the Problem of Agency. Journal of Critical Realism, 5(1).

Archer, M.S. (2003). Structure, Agency and The Internal Conversation. Cambridge : Cambridge University Press.

Archer M.S. (2004). Entre la structure et l'action, le temps. Revue de Mauss, 24, 329-350.

Bartoli, A., Mazouz, B., Kéramidas, O. et Larat, F (2011). Éthique et performance en management public, Revue française d'administration publique, 140.

Berger P.L. \& Luckmann T. (1986). La construction sociale de la réalité, Méridiens Klincksieck et cie., coll. Sociétés, Paris.

Bezes, P. (2005). Le renouveau du contrôle des bureaucraties. L'impact du New Public Management. Informations sociales, 126, 26-37.

Bhaskar, R. (1989). The possibility of naturalism, $2^{\text {nd }}$ edition, Harvester, Hemel Hempstead.

Bouquin, H. (2004). La notion de performance. Journée d'étude I.A.E. De Tours, 15/1/2004.

Brunsson, N. (1989). The Organization of Hypocrisy - Talk, Decisions and Actions in Organisations. New York : John Wiley and Sons.

Carassus, D., Baradat, C., \& Dupuy, E. (2011). La caractérisation du pilotage universitaire : des pratiques adaptées à leur nouveau contexte ? Actes du Colloque AIRMAP, Université de VersaillesSaint-Quentin en Yvelines, 29, 30 juin et 1er juillet.

Chatelain-Ponroy, S. (2008). Le contrôle de gestion dans des bureaucraties professionnelles non lucratives : Une proposition de modélisation, HDR en Sciences de Gestion, Paris : Université Paris Dauphine.

Chauvey, J.-N. (2010). Hypocrisie, déraison : les nouveaux leviers du contrôle ? Comptabilité Contrôle-Audit, 16, 33-52.

Christensen, G. (1998). Le désengagement de l'Etat : étude critique d'un problème administratif dans son contexte politique. Revue internationale des sciences administratives, 54(1). 
Cohen, M.D., March, J.G., Olsen, J.P. (1972). «A Garbage Can Model of Organizational Choice », Administrative Science Quaterly, 17(1).

Crozier, M. (1965). Le Monde des employés de bureau; résultats d'une enquête, menée dans sept compagnies d'assurances parisiennes. Paris : Seuil.

De Geuser, F. \& Fiol, M. (2003). «Faire face à des situations complexes : La blessure narcissique des managers ", in Moingeon, B. (Ed), La formation des cadres dirigeants. L'apport de la recherche. Paris : L'Harmattan.

De Visscher, C. (2004). Autorités politiques et haute administration : une dichotomie repensée par la NGP ? Revue Internationale de Politique Comparée, 11(2).

De Vaujany, F-X. (2005). De la pertinence d'une réflexion sur le management des objets et outils de gestion. In de Vaujany, F.X. (Eds) De la conception à l'usage: vers un management de l'appropriation des outils de gestion. Cormelles-le-Royal : Editions EMS.

De Vaujany, F-X., Grimand, A. (2005). Réflexion sur la place de la perspective appropriative au sein des sciences de gestion. In de la conception à l'usage: vers un management de l'appropriation des outils de gestion (Eds, de Vaujany, F.X.). Cormelles-le-Royal : Editions EMS.

De Vaujany, F.X. (2006). Vers un dépassement de la notion d'utilisateurs : l'apport de la théorie des conversations internes. Revue d'Interaction Homme-Machine, 7, 14-22.

De Vaujany F.X (2007). Évaluer la « valeur à l'usage » de l'informatique. Une architecture de tableau de bord. Revue française de gestion, 173, 31-46.

Drucker, P. (1954). The Practice of Management. New York : Harper and Row.

Dubet, F. (2002). Le déclin de l'institution, Paris :Seuil, coll., « L'épreuve des faits ».

Dupuy, F. (1998). Le client et le bureaucrate. Paris : Dunod.

Emery, Y. \& Giauque, D. (2005). Paradoxes de la gestion publique. Paris : l'Harmattan.

Giauque, D. (2003). La bureaucratie libérale. Paris : l'Harmattan.

Giddens, A. (1984). The constitution of society: outline of a theory of structuration. Berkeley : University of California press.

Granget, L. (2009). Les universités en quête de prestige dans le grand jeu de la concurrence : le rôle de la communication marketing et l'impact des palmarès. Communication et organisation, 35, 148157.

Green, S. (2010). Valeur sûre. Réflexions sur l'argent et la morale dans un monde incertain. Paris : Éditions Collège des Bernardins.

Grimand A. (2006). Quand le knowledge management redécouvre l'acteur: la dynamique d'appropriation des connaissances en organisation. Management \& Avenir, 9,141-157.

Hatchuel, A. \& Weil, B. (1992). L'expert et le système. Paris : Economica.

Hofstede, G. (1978). The Poverty of Management Control Philosophy, Academy of Management Review, Juillet, 450-461.

Hofstede, G. (1981). Management Control of Public and Not-for-profit Activities. Accounting, Organizations and Society, 6(3), 193-211.

Hood, C.C. (1991). A Public Management for all Seasons ? Public Administration, 69(1), 3 - 20

Jacot, H. \& Fouquet, A. (2007). Le citoyen, l'élu, l'expert. Paris : Editions L'Harmattan. 
Lascoumes, P. et Simard, L. (2011). L'action publique au prisme de ses instruments. Revue française de science politique, 61(1), 5-22

Lauffer, R. Burlaud A. (1980). Management public : Gestion et Légitimité. Paris : Dalloz.

Lévesque, B (2012). La nouvelle valeur publique, une alternative à la nouvelle gestion publique? Revue Vie économique, 4(2).

Lorino, P. (1995). Comptes et Récits de la Performance - Essai sur le pilotage de l'Entreprise. Paris : Les Editions d'Organisation.

Lorino, P. (2002). Vers une théorie pragmatique et sémiotique des outils appliquée aux instruments de gestion. Document de recherche ESSEC, DR 02015.

March, J.G. (2006). Rationality, foolishness, and adaptative intelligence. Strategic Management Journal, 27,pp. 201-214.

Marquet P. \& Leroy, F. (2004). Comment conceptualiser les usages pédagogiques des environnements numériques de travail et d'apprentissage partagés. 7e Biennale de l'éducation et de la formation, Lyon, France. Consulté le 20 mai 2013 sur le site: www.inrp.fr/biennale/7biennale/Contrib/longue/3094.pdf

Martinet, A.C. (2008). La gestion publique à l'ère de l'institutionnalisation des entreprises et de la managérialisation des institutions publiques. Un réexamen axiologique et épistémologique. In Mazouz, B., le métier de gestionnaire public à l'aune de la gestion par résultats. Sainte-Foy: Presses de l'Université du Québec

Mazouz, B. \& Coté, L. (2005). Les effets de la loi sur l'administration publique sur la qualité des services et sur la gestion dans les ministères et les organismes. Québec : Ecole Nationale d'administration publique.

Mazouz, B., Garzon, C. \& Picard, P. (2012). Les déviances dans les organisations publiques en quête de performance : vers une gestion prophylactique des risques de déviance. Management international, 16(3), 92-100.

Mevellec P. (1990). Outils de gestion : la pertinence retrouvée. Paris: Éditions Comptables Malesherbes.

Mignot-Gérard,S.(2008). Gouvernance des universités. In VanZanten, A. (dir.), Dictionnaire de l'Éducation, Paris : PUF.

Mintzberg, H. (1990). Le management : voyage au centre des organisations. Paris : Éditions d'Organisation.

Moisdon J.C. (1997). Du mode d'existence des outils de gestion. Paris: Séli-Arslan.

Morin, E. (2007). Restricted Complexity, General Complexity. In Gershenson, C., Aerts D., Edmonds, B.(Eds.), Worldviews, Science and Us, Philosophy and Complexity (pp. 5-29). Londres : World Scientific.

Musselin, C. (1997). Les universités sont-elles des anarchies organisées? In Chevallier J. (Ed.). Désordre(s), (pp. 67-94). Paris : P.U.F., CURAPP.

Musselin C. (2001).La longue marche des universités françaises. Paris : PUF.

Paradeise, C (2012). Le sens de la mesure. La gestion par les indicateurs est-elle gage d'efficacité ? Revue d'économie du développement, 26, 67-94.

Pesqueux, Y. (2003). La notion de performance globale. hal.archives-ouvertes.fr 
Pettigrew, A.M. (1997). What is a processual analysis. Scandinavian Journal of Management, 13, 337-348.

Pfeffer, J.\& Sutton, R. (2006). Hard facts, Dangerous half-truths and total nonsense. Profiting from evidence-based management. Boston : Harvard Business School Press.

Rabardel, P. (2005). Instrument, activité et développement du pouvoir d'agir. In P. Lorino, \& R. Teulier (Ed.), Entre connaissance et organisation : l'activité collective (pp. 251 -265). Colloque de Cerisy. Paris : La découverte.

Reynaud, J.D. (2003). Régulation de contrôle, régulation autonome, régulation conjointe. In De Terssac G. (éd.), La théorie de la régulation sociale de Jean-Daniel Reynaud (pp. 103-113). Paris : La Découverte.

Simons, R. (1995). Levers of control: how managers use innovative control systems to drive strategic renewal. Boston : Harvard Business School Press.

Urio, P. (1998). La gestion publique au service du marché. In M. Hufty, La pensée comptable : État, néolibéralisme, nouvelle gestion publique. Les nouveaux Cahiers de l'IUED (pp. 91-124). Paris et Genève : PUF.

Vinokur, A. (2008). La loi relative aux libertés et responsabilités des universités : essai de mise en perspective. Revue de la régulation, 2.

$\mathrm{W}$

e

i

$\mathrm{c}$

$\mathrm{k}$

,

K

E 